\title{
Homöopathie und Psychotherapie bei der Behandlung von Angsterkrankungen und Depressionen
}

Homöopathie ist gut geeignet, eine psychotherapeutische Behandlung zu begleiten - Akute Beschwerden und Krisen werden gemildert, der Leidensdruck verringert

\section{Zusammenfassung}

Psychotherapie und Homöopathie können in der Behandlung von Angsterkrankungen und Depressionen gut kombiniert werden. Homöopathie kann ähnlich wirken wie eine psycho-pharmakologische Behandlung: beruhigend und lindernd bei akuten Beschwerden und Krisen. Die psychisch-emotionale Erlebnisfähigkeit bleibt bei homöopathischer Begleitbehandlung erhalten.

Anhand von 3 Fallbeispielen wird gezeigt, bei welchen Indikationen sich die Kombinationstherapie eignet und wie ein geeignetes Setting aussehen kann.

Angsterkrankungen und Depressionen können sehr gut mit einer Kombination von Homöopathie und Psychotherapie behandelt werden, wenn Indikationen, Kontraindikationen und ein sorgfältig ausgewähltes Setting beachtet werden.

\section{Indikationen und Kontraindikationen}

Indiziert ist die gleichzeitige Behandlung mit beiden Methoden bei depressiven Erkrankungen wie Dysthymie, Anpassungsstörungen oder depressiven Episoden und phobischen oder diffusen Angststörungen. Auch posttraumatisch bedingte Angst oder depressive Symptome sprechen gut auf diese Behandlungskombination an.

Eine Kontraindikation besteht bei den meisten psychotisch bedingten Ängsten oder Depressionen, ebenso bei Suchterkrankungen oder akuter Suizidalität.

\section{Setting}

Bei passender Indikation ist dann das geeignete Setting zu wählen. Zuerst ist es wichtig zu klären, ob die homöopathisch-psychotherapeutische Behandlung von einem oder zwei Therapeuten durchge-

führt werden soll. Dafür muss das strukturelle Niveau des Patienten diagnostiziert werden.

Depressive und Angstsymptome können sich nämlich vor dem Hintergrund eines reifen oder unreifen psychischen Strukturniveaus entwickeln. Aber nur Patienten, die eine reifere innere Entwicklungsstufe erreicht haben, können die zwei Rollen, die ein und derselbe Behandler als Homöopath UND Psychotherapeut einnimmt, unterscheiden. Patienten mit einer unreifen BorderlineStruktur bspw. werden mögliche Erstverschlimmerungen, die sich bei einer homöopathischen Behandlung ergeben können, dem Behandler zuschreiben und ihm die Schuld daran geben, dass es ihnen schlechter geht. Sie werden dann im schlimmsten Fall die Behandlung abbrechen. Diese Patienten können die Rollen nicht unterscheiden und müssen von zwei unterschiedlichen Therapeuten behandelt werden.

Bei neurotischen Patienten mit einem reiferen Strukturniveau kann der homöopathische Behandler gleichzeitig auch der psychotherapeutische sein, denn sie sind in der Lage, Rolle und Person zu unterscheiden.

\section{Behandlungssituationen}

Eine kombinierte homöopathisch-psychotherapeutische Behandlung kann auf verschiedenen Wegen zustande kommen.

Die eine Möglichkeit ist, dass der Patient sich ganz bewusst einen Therapeuten auswählt, der beide Methoden anwenden kann. 


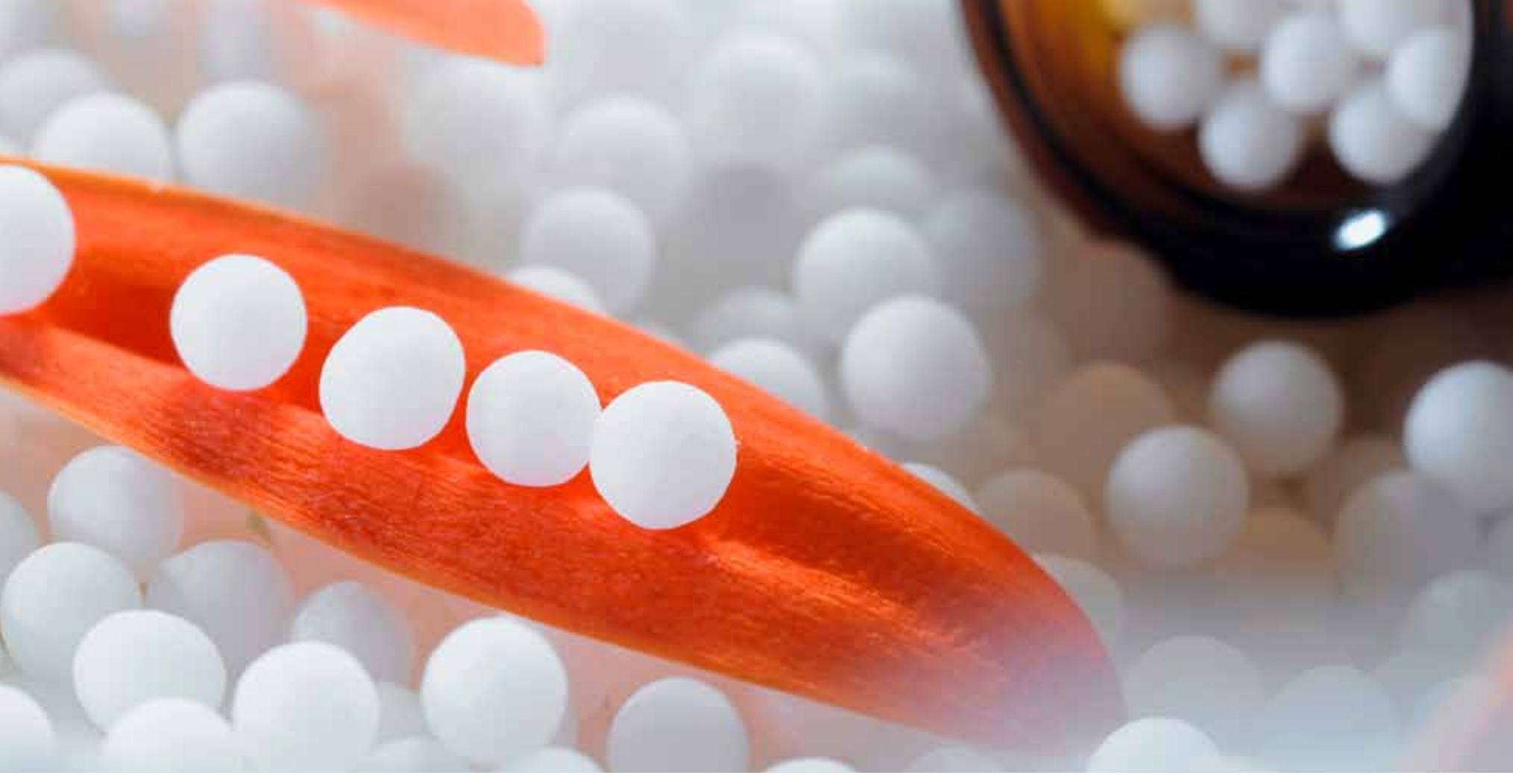

Die integrierten Versorgungsverträge, die der Deutsche Zentralverein homöopathischer Ärzte (DZVhÄ) mit verschiedenen Krankenkassen (KK) abgeschlossen hat, ermöglichen Patienten diese Wahl, denn die beteiligten KK erstatten sowohl die psychotherapeutische als auch die homöopathische Behandlung. Der Therapeut muss allerdings ein Kassenarzt sein, der auf einer Liste der Krankenkassen geführt wird. Bei privaten KK ist es je nach Vertrag des Patienten ebenfalls möglich, beide Verfahren kombiniert anzuwenden.

\section{Dazu ein Fallbeispiel}

Die junge Frau sucht mich auf, nachdem sie gezielt im Internet recherchiert hatte. Sie war extra zu einer Krankenkasse gewechselt, die sowohl die homöopathische wie die psychotherapeutische Behandlung übernimmt. Nach qualvollen Jahren voller Angst, Depressionen und Schmerzen und vergeblicher allopathischer Therapie wollte sie jetzt eine Kombinationstherapie mit den beiden Behandlungsmethoden versuchen.

\section{Symptomatik}

Seit einem schweren Autounfall vor 5 Jahren leidet sie an Panikattacken: Sie bekommt Luftnot, Herzrasen, Schweißausbrüche und große Angst, vor allen Dingen in engen Räumen und beim Alleinsein. Zusätzlich dazu traten vor 3 Jahren plötzlich linksseitige Schmerzen an Beinen und Armen auf, die nach rechts wandern. Sie kann sich dann nur noch eingeschränkt bewegen und verbringt Tage verzweifelt im
Bett! Morgens beim Erwachen ist es immer am schlimmsten, sowohl die Schmerzen als auch die Angst, aber auch wenn sie lange schläft wird alles schlimmer. Sie ist sehr deprimiert, fühlt sich hoffnungslos, hat keinen Antrieb mehr und kann sich nicht konzentrieren.

Natürlich war alles medizinisch abgeklärt, ohne jeglichen organpathologischen Befund. Die psychiatrischen Kollegen verschrieben ihr nur immer neue Kombinationen von Psychopharmaka, ohne dass sich etwas gebessert hatte.

Die Symptomatik entwickelte sich nach einem Autounfall, der passierte, als sie auf dem Weg zu ihrer Mutter war. Die Mutter ist schwer depressiv und die Tochter fährt jedes Wochenende über 1000 Kilometer, um sich um die Mutter zu kümmern.

\section{Diagnose}

Die Patientin leidet also an einer Panikstörung (F 41.0), einer anhaltenden somatoformen Schmerzstörung (F 45.4) und einer mittelgradig depressiven Episode (F 32.1). Definition s. Beitrag S.44.

\section{Therapie}

Es wird ein Antrag auf Kurzzeittherapie für 25 Stunden gestellt und eine homöopathische Erstanamnese durchgeführt. Das Arzneimittel Lachesis erfasst ihre Symptomatik am besten und wird ihr in einer Hochpotenz verordnet. Daraufhin verschwinden die Körperschmerzen und Panikattacken innerhalb von 4 Wochen. Gleichzeitig wird in der psychotherapeutischen Behandlung ihr 
unbewusster Autonomie-Abhängigkeits-Konflikt bearbeitet: Sie fühlte sich als einzige Tochter ein Leben lang für ihre kranke Mutter verantwortlich und konnte deshalb ihre Autonomie nur unvollständig und mit großen Schuldgefühlen entwickeln. In der Therapie lernt sie, sich mit der Mutter auseinanderzusetzen und sich von ihr abzugrenzen, um ein selbstständiges Leben führen zu können.

Die homöopathische Behandlung linderte also ihre psychische und psychosomatische Symptomatik schnell und nachhaltig und die gleichzeitig stattfindende Psychotherapie ermöglichte es ihr, den unbewussten Konflikt so zu lösen, dass sie nicht mehr symptomatisch werden musste.

Sechs Monate nach Beendigung der Therapie erhielt ich einen Anruf der überglücklichen jungen Frau: Sie hatte geheiratet und war endlich schwanger! Seit Jahren wollte sie schon ein Kind und nun hatte es endlich geklappt!

\section{Homöopathie und Psychotherapie}

\section{ergänzten sich in diesem Fall so}

\section{gut, dass eine Angststörung und}

\section{Depression innerhalb eines halben}

\section{Jahres geheilt werden konnte.}

Die zweite Möglichkeit, eine psychotherapeutische mit der homöopathischen Behandlung zu kombinieren, ergibt sich während einer laufenden Psychotherapie. In der psychotherapeutischen Behandlung können Symptome und Krisen auftreten, die durch ein homöopathisches Arzneimittel gelindert und beherrscht werden können, wie folgendes Beispiel zeigt:

\section{Fallbeispiel 2: Behandlung von Krisen}

Eine sehr engagierte Erzieherin war schon seit einigen Monaten wegen einer depressiven Erkrankung in Psychotherapie. In dem Hort, wo sie arbeitete, war sie mit einer Kollegin im Konflikt. Diese Kollegin kam eines Tages in ihre Gruppe und beschuldigte einige Kinder grundlos des Diebstahls. Die Patientin versuchte, sich schützend vor ihre Kinder zu stellen, was ihr aber nicht gelang: Die Kollegin schrie herum und schlug ein Mädchen.

Die Patientin meldete diesen Vorfall, und es gab ein Disziplinarverfahren gegen die Kollegin. Nach dem Ereignis entwickelte sie aber Belastungssymptome und war nicht mehr in der Lage, an ihren Arbeitsplatz zurückzukehren. „Ich fühle mich starr, gehetzt, ich kann mich nicht mehr stillhalten und muss ununterbrochen gehen. Ich zittere, habe Angstgefühle und mir ist eiskalt, ich friere ständig und dann bin ich wieder kaltschweißig. Mein Herz klopft mir bis zum Hals. Es ist schrecklich, ich bin in einem Alarmzustand!“

\section{Therapie}

Die Patientin hatte noch nie vorher eine homöopathische Behandlung gehabt. Ich schlug ihr vor, ein homöopathisches Arzneimittel zu nehmen, um sich zu beruhigen.
Ich gab ihr Aconit C $\mathbf{1 0 0 0}$ in der Praxis, und schon auf dem Heimweg spürte sie Erleichterung: „Meine Hände wurden warm“, so berichtete sie in der Therapiestunde eine Woche später, „mein Herzschlag normalisierte sich und ich hörte auf zu zittern. Das Zittern ist nämlich mein Leiden: Wenn es mir irgendwie schlecht geht, fange ich so sehr an zu zittern, dass ich keine Tasse mehr halten kann! Ich bin so froh, dass es jetzt vorbei ist und ich auch nicht mehr diese Eiseskälte spüre, sondern wieder warm bin, auch innerlich! Außerdem habe ich keine Angst mehr und fühle mich nicht so getrieben, ich muss keine stundenlangen Spaziergänge mit meinen Hunden machen und kann mich wieder ruhig hinsetzen."

Nachdem die akute Symptomatik abgeklungen war, konnten wir therapeutisch weiterarbeiten und versuchten $\mathrm{zu}$ verstehen, warum sie so dramatisch auf den Vorfall mit ihrer Kollegin reagiert hatte.

Ein Telefonat mit ihrer Schwester gab den Schlüssel dazu: „Meine Schwester, der ich es erzählt hatte, sagte zu mir: ,Kein Wunder, dass Du so reagierst, weißt Du es nicht mehr: Du konntest mich genauso wenig vor den Schlägen des Vaters beschützen, wie Du jetzt Deine Kinder vor den Schlägen der Kollegin schützen konntest! ““

Nun erinnerte sich die Patientin, dass sie als Kind immer versucht hatte, ihre kleine Schwester vor den brutalen Misshandlungen des Vaters zu schützen. Er ging wegen kleinster Verfehlungen auf seine Töchter los, bevorzugt jedoch auf die Jüngste. Dann schlug er sie zu Boden und trat mit den Stiefeln nach ihr - er war Polizist. Als erwachsene Frau zeigte die Schwester dann so auffällige neurologische Symptome, dass eine Magnetresonanztomografie gemacht wurde und alte Hirnblutungen festgestellt wurden - eine Folge der damaligen Misshandlungen.

„Ich wollte mich immer vor meine Schwester stellen, es gelang mir aber nicht, denn dann schlug mein Vater mich auch. Ich hatte Todesängste und Schuldgefühle, es war schrecklich, das Martyrium dauerte meine ganze Kindheit, niemand hat geholfen, obwohl es viele wussten, wie uns der Vater misshandelte! “, so erzählte die Patientin weiter. Endlich konnte sie weinen, aber auch die Wut auf den brutalen Vater spüren.

Das Arzneimittel half in dieser Situation, die Angstsymptomatik aufzulösen, sodass wir psychotherapeutisch weiterarbeiten konnten. Der Vorfall mit der Kollegin hatte ihr kindliches Trauma angetriggert und sie entwickelte ein Posttraumatisches Belastungssyndrom. Das homöopathische Mittel beruhigte die Patientin so gut, dass sie sich in der Psychotherapie an die Traumatisierung erinnern und sie aufarbeiten konnte.

\section{Fallbeispiel 3: Konstitutionelle Therapie bei Depression}

Angstsymptome sprechen oft sehr gut auf ein passendes homöopathisches Arzneimittel an, das aber trotzdem individuell verordnet werden muss. In der Homöopathie gibt es keine indikationsbezogenen Standardmittel. Angsterkrankungen können also eher symptomatisch behandelt werden, während Depressionen meist nach einer konstitutionellen homöopathischen Therapie verlangen, wie folgendes Beispiel zeigt:

\section{Symptomatik}

Die schüchterne junge Frau war schon seit 3 Jahren wegen einer schweren depressiven Erkrankung in psychoanalytischer Therapie. 
Sie war in ihrer Kindheit Vernachlässigung und Gewalt ausgesetzt gewesen: ihre Mutter lehnte sie ab, weil sie das ungewollte dritte Kind war, und der Vater misshandelte sie. Sie bekam so wenig Liebe und Geborgenheit, dass sie sich zu den Schafen in den Stall legte, um etwas Wärme zu spüren!

Das alles wurde ihr in der Psychotherapie zunehmend bewusst, und obwohl sie sich durch die therapeutische Behandlung zwischenzeitlich gut stabilisiert hatte, drohte sie nun wieder zu dekompensieren. Sie konnte nur noch mühevoll ihrer Arbeit nachgehen, sonst aber das Haus nicht verlassen. Sie konnte sich nicht mehr ausreichend versorgen, lebte von Eiern, Milch und Brot und hatte alle sozialen Beziehungen abgebrochen.

„Ich bin ständig so müde“, klagte sie weinend „es stört mich alles und ich bin gereizt. Innerlich bin ich zwar sehr unruhig, aber ich kann mich nicht bewegen, sondern liege nur in meiner Wohnung. Ich kann wieder nicht schlafen, nächtelang liege ich wach. Jede kleinste Anstrengung erschöpft mich so sehr, dass ich mich nach dem Treppensteigen - und es ist nur ein Absatz bis zu meiner Wohnung - schon wieder hinlegen muss. Ich kann mich auch nicht konzentrieren, mache Fehler beim Schreiben und stopfe Süßigkeiten in mich hinein, aber dann geht es mir noch schlechter!“

Auf meine besorgte Frage, ob sie denn auch daran denke, sich das Leben zu nehmen, schüttelt sie den Kopf. „Um mich aktiv umzubringen, hätte ich gar nicht die Kraft! Aber wenn ich einfach so sterben würde, würde ich es begrüßen! Mein Leben kommt mir einfach so sinnlos vor. Ich werde nie mehr gesund werden, nichts macht mir mehr Freude!“

\section{Therapie}

In solch einem schlechten Zustand brauchte die junge Frau entweder ein Antidepressivum oder ein homöopathisches Arzneimittel. Ich hatte bei einer anderen Gelegenheit früher schon einmal ihr Konstitutionsmittel repertorisiert und gab ihr deshalb 3 Globuli Calcium carbonicum C 1000.

Eine Woche später kommt die Patientin zur Sitzung und berichtet strahlend, dass es ihr gut gehe: „Gleich nach der letzten Stunde habe ich ganz schlimme Kopfschmerzen gekriegt, wie früher schon immer, und ich war sehr müde. Ich musste mich dann zu Hause hinlegen und habe 10 Stunden geschlafen, wie schon seit Wochen nicht mehr! Als ich aufwachte, habe ich mich erholt gefühlt. Ich habe das die letzten Wochen verlernt: zu schlafen, um mich erholt zu fühlen! In den letzten Wochen habe ich die Müdigkeit gar nicht mehr gespürt, jetzt wurde mir klar, dass ich müde bin. Ich kannte dieses Gefühl einer gesunden Müdigkeit gar nicht mehr. Ich wollte mich hinlegen und schlafen. Das war nicht die erregte Erschöpfung wie die letzten Wochen, sondern eine echte Müdigkeit, und es ist so schön, diese Müdigkeit wieder zu spüren! Seither geht es mir gut, ich möchte nicht mehr sterben, sondern gehe durch die Stadt und finde die Welt um mich herum schön. Ich kann mich wieder konzentrieren, habe jetzt auch meine Wohnung aufgeräumt und das Geschirr abgewaschen, das seit Wochen schmutzig herumstand!“

Nach dieser Krise konnten wir die Psychotherapie fortsetzen und die Patientin entwickelte sich sehr gut. Im folgenden Therapieverlauf half ihr das Konstitutionsmittel immer wieder, wenn sie depressiv zu werden drohte. Sie brauchte trotz ihrer schweren Symptomatik kein Antidepressivum.

\section{Fazit}

Die Homöopathie kann in Kombination mit Psychotherapie in der Therapie von Depressionen und Angsterkrankungen eine echte Alternative zur psychopharmakologischen Behandlung darstellen.

Im Gegensatz zur allopathischen Behandlung erleben die Patienten durch die homöopathischen Arzneimittel keine Beeinträchtigung ihres emotionalen Erlebens. Antidepressiva dämpfen oft die innere Wahrnehmung und Schwingungsfähigkeit, die Patienten fühlen sich stumpf, wie in Watte gepackt, sich selbst entfremdet, und das erschwert den therapeutischen Prozess. Eine begleitende homöopathische Behandlung beruhigt krisenhafte Situationen während einer Psychotherapie, lindert psychische und psychosomatische Symptome und ermöglicht eine Fortführung der Therapie, wenn schmerzhafte Erinnerungen die Patienten zu überwältigen drohen. Die Homöopathie kann ähnlich wirken wie eine psychopharmakologische Behandlung, jedoch ohne die psychisch-emotionale Erlebnisfähigkeit zu beeinträchtigen.

Das erhöht die Compliance und das Gefühl der Selbstbestimmung. Viele Patienten wehren sich gegen Psychopharmaka, weil sie sich dadurch fremdbestimmt fühlen. Gerade diese Patienten suchen die Möglichkeit nach einer kombinierten Therapie, weil sie ihre Krankheit verstehen und von innen heraus heilen wollen. Dazu sind die beiden Methoden in ihrer Kombination sehr gut geeignet

Interessenkonflikt: Der Autorin erklärt, dass keine wirtschaftlichen oder persönlichen Verbindungen bestehen.

Online zu finden unter

http://dx.doi.org/10.1055/s-0034-1396351

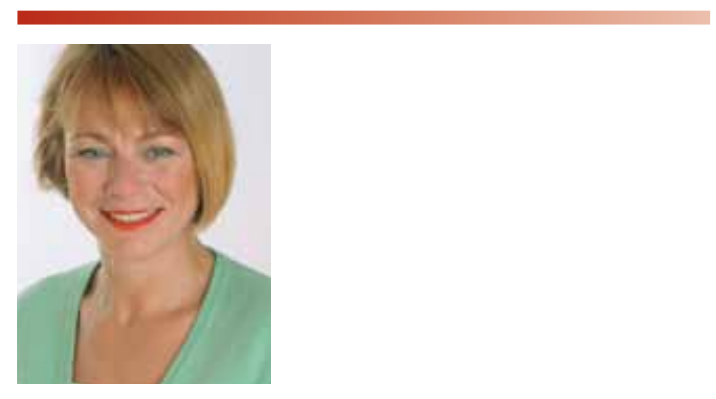

Dr. med. Ingrid Pfanzelt

Fachärztin für Psychosomatische Medizin und Psychotherapie, Psychoanalyse, Homöopathie

St. Anna Platz

880538 München

praxis@dr-pfanzelt.de

Studium der Medizin in Perugia/Italien und an der LMU München, Dissertation in der Kinder- und Jugendpsychiatrie. Facharztausbildung in Psychosomatischer Medizin und Psychotherapie, Ausbildung zur Psychoanalytikerin, Ausbildung in Traumatherapie (EMDR), Balint-Gruppen-Leiterin (BLÄK). Erste homöopathische Vorlesungen besuchte sie schon während des Studiums bei Willibald Gawlik und Arthur Braun. Ihre homöopathische Weiterbildung beendete sie 1993, sie erwarb das Homöopathie-Diplom des DZVhÄ. Seit 1993 ist sie niedergelassen in eigener Kassenpraxis für Psychotherapie und Homöopathie. 